\title{
Nécessité, genèse et vie d'une réglementation des actions sur les cours d'eau
}

\author{
Necessity, genesis and life of regulations \\ of actions on waterways
}

\author{
Marie-Edith Zamuth \\ Institut de Droit de l'Environnement, Lyon
}

\begin{abstract}
"Les problèmes que posent l'utilisation de l'eau pour l'alimentation de l'homme et du bétail, la culture, la navigation, l'utilisation de la force motrice sont d'une importance exceptionnelle. Les conditions de vie d'une région, son développement économique sont sous la dépendance du régime des eaux. Aussi depuis longtemps a-t-on restreint les droits des propriétaires dans l'intérêt de la communauté ; les eaux sont de plus en plus considérées comme un richesse nationale collective et l'on est loin du point de départ formé par le droit romain qui voyait dans les eaux l'objet d'une propriété privative " (Alex Weill, Droit Civil - les Biens, Dalloz, 1970, $\left.\mathrm{n}^{\circ} 5\right)$.
\end{abstract}

Depuis la Révolution, le droit d'usage des eaux, et notamment des rivières, a fait l'objet d'une grande attention de la part du législateur et du pouvoir réglementaire.

Les pouvoirs publics se sont vus reconnaitre des compétences toujours plus étendues pour organiser la répartition de la ressource entre les différents usages, privés ou publics, et la satisfaction des intérêts généraux tels que la salubrité publique. Celle-ci est traditionnellement assurée par la multiplication des moyens contraignants, caractéristiques des polices administratives: la légalité d'exercice d'une activité déterminée est subordonnée à une autorisation ou à une déclaration.

La menace pesant sur ces intérêts généraux justifie aussi les modifications et, le cas échéant, le retrait des décisions individuelles.

Comme toutes les autres utilisations de l'eau, la réglementation de l'édification d'ouvrages dans les cours d'eau s'est progressivement renforcée.

- Après avoir défini les critères juridiques des cours d'eau domaniaux - à l'époque navigables ou flottables et des cours d'eau non domaniaux - ou non navigables ni flottables - , distinction déjà établie par le droit romain et consacrée par le code civil (articles 538 et 644 ), la loi du 8 avril 1898 sur le régime des eaux, considérée comme une véritable charte de l'eau, pose les règles de l'implantation et le fonctionnement des ouvrages hydrauliques.

Presque entièrement codifiée - dans le code rural pour les cours d'eau non domaniaux et dans le code du domaine public fluvial et de la navigation intérieure pour les cours d'eau domaniaux - ce texte n'a fait que reprendre solennellement les principes établis depuis 1789 et constituant la réglementation actuelle en la matière. Elle soumet à autorisation administrative tout ouvrage, avec ou sans prise d'eau, situé dans le lit d'un cours d'eau.

L'innovation de 1898 a été de réglementer l'ensemble des usages de l'eau, quelle que soit leur finalité, traditionnelle comme l'agriculture ou plus récente comme l'industric.

- La loi du 16 octobre 1919 relative à l'utilisation de l'énergie hydraulique - modifiée par la loi 80-531 du 15 juillet 1980 relative aux économies d'énergie et à l'utilisation de la chaleur - met en place un régime spécifique pour la production hydroélectrique : toute installation doit être autorisée ou concédée par l'Etat, seul compétent pour gérer cette nouvelle richesse nationale.

- La loi du 16 décembre 1964 n'a apporté aucun changement à la réglementation des ouvrages hydrauliques. Elle a introduit de nouvelles notions dont certaines ne connaîtront aucune application : il en sera ainsi notamment pour le débit réservé, le débit affecté et les cours d'eau mixtes.

- La loi du 29 juin 1984 relative à la pêche en eau douce et à la gestion des ressources piscicoles va assortir de sanctions pénales spécifiques les dispositions relatives à la police des cours d'eau et dont le non-respect était, jusquelà, puni par de simples peines contraventionnelles peu dissuasives.

En outre, elle renforce les obligations auxquelles sont assujettis la construction et le fonctionnement des ouvrages 
hydrauliques afin de préserver la faune piscicole - dont la protection est reconnue d'intérêt général - et étend théoriquement les pouvoirs de la police des eaux en matière de modification et de suppression des droits d'eau existants.

L'Administration qui était traditionnellement chargée d'assurer le libre écoulement des eaux doit aussi garantir la conservation des milieux aquatiques.

- Une autre limitation à l'usage des eaux par les particuliers est issue de la loi du 22 juillet 1987 relative à l'organisation de la sécurité civile, à la protection de la forêt contre l'incendie et à la prévention des risques majeurs. En cas de non-respect de la réglementation relative aux ouvrages hydrauliques, l'autorité de police peut prononcer des sanctions administratives analogues à celles existant en matière d'installations classées. De plus, les exploitants d'ouvrages implantés dans le lit d'un cours d'eau pourront être temporairement tenus, sous certaines conditions, à des obligations exorbitantes en matière de fonctionnement des ouvrages et de débit réservé dès lors qu'une sécheresse grave menace l'alimentation en eau des populations.

Les principes posés en 1898 n'ont donc jamais été remis en cause et le Parlement n'a légiféré que pour étendre et renforcer les pouvoirs de l'autorité chargée de la police des eaux afin de sauvegarder des intérêts généraux dont la multiplication va de pair avec l'émergence de nouvelles aspirations sociales.

Ainsi, les différentes actions entreprises dans le lit d'un cours d'eau et sur ses berges vont être étroitement contrôlées par l'administration.

Le recours à la réglementation apparaît comme le moyen d'éviter les conflits d'intérêts soit en définissant, pour un usage déterminé, les règles opposables à tous, soit en privilégiant la satisfaction d'intérêts, jugés supérieurs, au détriment d'intérêts privés (compensés, le cas échéant, par une indemnisation).

La nécessité de réglementer s'est, semble-t-il, imposée au législateur chaque fois qu'un besoin nouveau se manifestait ou plus exactement qu'un aspect existant prenait une intensité nouvelle.

Le "droit des cours d'eau " n'échappe pas à ce phénomène et il suffit d'essayer de dresser la liste des dispositions traitant directement ou indirectement de la question.

Différentes raisons expliquent cette complexité.

Tout d'abord, le droit de l'eau est formé de nombreux textes dont l'objet est spécifique : la loi de 1919 sur l'énergie hydroélectrique, le décret-loi de 1935 instituant les plans de surfaces submersibles, etc. A chaque type d'intervention sur un cours d'eau, un texte spécial.

Bien qu'incomplet, le travail de codification effectué dans le code rural et dans le code du domaine public fluvial et de la navigation intérieure facilite la lecture de ces dispositions, multiples et dispersées, applicables aux ouvrages hydrauliques et à l'entretien des cours d'eau.

De plus, les problèmes posés par l'eau ne concernent pas que le droit de l'eau, domaine juridique particulier, mais ont des incidences dans les multiples branches du droit, tout spécialement la santé publique, l'urbanisme, les transports et l'aménagement du territoire. Il suffit pour s'en convaincre de feuilleter les différents codes.

Une autre cause de complexité vient des caractéristiques physiques de l'élément, objet de droit. En effet, l'eau des cours d'eau est un élément fluide qui s'écoule ; elle engendre des solidarités de fait entre les différents utilisateurs. Or le droit a toujours des difficultés pour appréhender globalement les effets liés à ce caractère : contournant l'obstacle, la réglementation a procédé par la compartimentation des eaux et de leurs utilisations, ne tenant pas, ou peu, compte de l'interdépendance des différents usages et les divers interêts qui se manifestent tout au long d'un cours d'eau, voire dans tout son bassin versant.

Dans cette optique particulière, l'aménagement des cours d'eau apparaît comme la succession d'interventions indépendantes les unes des autres.

On est donc loin de la réalité qui imposerait une gestion intégrée : l'aménagement du cours d'eau ne peut pas limiter aux ouvrages qui y sont établis mais il englobe aussi l'entretien du lit et des berges, la prévention contre les pollutions et les inondations, la gestion des nappes alluviales. De plus, il doit concilier différents intérêts souvent conflictuels tels que l'implantation d'un barrage, l'extraction de granulats, l'exercice de la navigation de loisirs ou la protection de l'environnement.

A l'heure actuelle, cet édifice juridique est remis en question car les solutions proposées par le droit, lorsqu'elles existent, ne sont plus satisfaisantes.

En effet, de nouvelles exigences sont apparues qui nécessitent une gestion globale du cours d'eau.

Il n'est plus possible d'ignorer l'existence du bassin versant dans la gestion d'une rivière et de se contenter de la juxtaposition de décisions ponctuelles.

Il n'est plus possible d'imposer unilatéralement la vision de l'administration centrale et de ne pas associer toutes les personnes directement intéressées par la question et tout particulièrement les collectivités territoriales.

Dès lors qu'on reconnaît à la rivière sa qualité d'écosystème, caractérisé par des interdépendances et des équilibres biologiques, il n'est plus possible de procéder à une gestion cloisonnée de chaque usage par des organismes spécialisés.

La loi de 1964 a été la première grande avancée en matière de gestion globalisée des cours d'eau : d'une part, elle va ériger juridiquement le bassin comme l'échelon nécessaire et adapté aux impératifs de gestion (agences financières de bassin, article 175 du code rural) et, d'autre part, elle préconise l'élaboration des décrets d'objectifs de qualité prenant ainsi en compte l'effet cumulatif des pollutions tout au long d'une rivière - ou tout au moins d'une section suffisamment autonome.

$\mathrm{Si}$ les agences financières de bassin ont prouvé, après plus de vingt ans de fonctionnement, leur efficacité, il n'en est pas de même de nombreuses dispositions de cette loi ; beaucoup sont restées lettre morte faute de la publication des décrets d'application. 
D'autres dont la mise en œuvre n'a pu être surmontée - comme les décrets d'objectifs de qualité — ont été remplacées par des pratiques administratives définies simplement par voie de circulaire (cartes départementales d'objectifs de qualité et contrats de rivières).

Réformer le droit de l'eau n'est pas une idée neuve mais toutes les tentatives ont abouti à des résultats très en-deçà de cette ambition.

On constate, en effet, que les mouvements "réputés" réformateurs se sont soldés par de simples «améliorations " visant a adapter le droit aux nouvelles exigences engendrées par le développement des besoins. Ainsi la loi du 8 avril 1898 n’a fait qu'entériner les dispositions élaborées au cours du XIX ${ }^{e}$ siècle.

Le droit de l'eau n'est, in fine, que le résultat d'une stratification progressive de textes dont l'enchevêtrement des dispositions rend difficile non seulement son appréhension mais aussi, ce qui est plus grave, son application.

Jusqu'alors aucun texte n'a eu la capacité intrinsèque de s'adapter aux évolutions. Le législateur a toujours dû intervenir pour modifier le dispositif juridique dont la rigidité ne constitue pas toujours un atout.

Depuis 1987, il est apparu nécessaire de réviser notre droit de l'eau. En effet, le système existant se révèle insuffisant voire lacunaire pour concilier les différents usages de l'eau dont le caractère conflictuel a été exacerbé par deux années de sécheresse mais aussi pour assurer une reconnaissance et une protection efficace du milieu aquatique.

L'adoption du plan national pour l'environnement a rendu plus urgente encore cette réforme.

L'avant-projet de loi déposé fin mars, pour avis devant le Conseil d'Etat résulte d'un long travail de réflexion fondé sur une large consultation des nombreux acteurs intervenant dans le domaine de l'eau (groupe national de l'eau, assises régionales à l'échelon de chaque bassin et assises nationales de l'eau qui se sont tenues à Paris les 20 et 21 mars 1991).

Si le ministère de l'Environnement a progressivement allégé le texte en lui soustrayant les dispositions relatives à la mer, au rôle des agences financières de bassin et au curage des cours d'eau non domaniaux, on peut toutefois noter la permanence de quelques idées directrices et novatrices.

\section{La nécessité d'une gestion équilibrée et solidaire imposée par l'unité de la ressource et l'interdépen- dance des différents besoins et usages, et afin de concilier les exigences de l'écologie et de l'économie}

\subsection{La volonté de gestion globale transparaît}

1.l.l. dans la globalisation et l'élargissement du champ d'application de la loi de 1964 (modification de l'article $1^{\mathrm{er}}$ ). a. A côté des objectifs traditionnels (lutte contre la pollution des eaux et répartition de la ressource en vue de satisfaire les différentes activités humaines - alimentation en eau, lutte contre les inondations, agriculture, industrie, production d'énergie, transports, tourisme et loisirs... s'exerçant légalement), la préservation des écosystèmes aquatiques, des sites et des zones humides devient une des préoccupations du droit de l'eau. Cette évolution s'inscrit dans le prolongement des lois de 1976 et 1984.

De plus, il y a une reconnaissance de la valeur des écosystèmes aquatiques et notamment des zones humides.

Par exemple, les « nouveaux » plans de surfaces submersibles devraient avoir pour objectif d'assurer la conservation du fonctionnement des écosystèmes que constituent les champs d'inondation.

b. La nouvelle loi devrait aussi s'appliquer à tout fait susceptible d'avoir une incidence sur le volume, le régime, le mode d'écoulement ou la qualité physique, chimique, biologique ou microbiologique des eaux et des milieux aquatiques alors que la loi de 1964 ne vise que les faits susceptibles de provoquer ou d'accroître la dégradation des eaux.

De nombreuses lacunes du droit de l'eau seront ainsi comblées puisque pourront être réglementés notamment les prélèvements dans les cours d'eau non domaniaux, la création de retenues collinaires ou d'étangs, tous les captages dans les eaux souterraines.

De plus, tous les déversements, écoulements, rejets ou dépôts sont concernés, qu'ils soient chroniques ou épisodiques, ou même non polluants.

1.1.2. dans la mise en place d'une planification au niveau du bassin (dont l'importance est réaffirmée en matière de gestion des eaux), voire du sous-bassin.

Arrêté par l'Etat après consultation des différents acteurs dans le domaine de l'eau (conseils généraux et comités de bassin intéressés), le schéma d'aménagement et de gestion des eaux (ou SAGE) a deux objets :

- d'une part, fixer les objectifs généraux d'utilisation, de mise en valeur et de protection quantitative et qualitative de la ressource en eau et du fonctionnement des écosystèmes aquatiques ;

- et, d'autre part, prendre en compte, orienter, et le cas échéant, harmoniser les documents d'orientation ou de planification (cartes départementales d'objectifs de qualité, schémas d'aménagement des eaux, contrats de rivières, schémas départementaux de vocation piscicole...) et les programmes des personnes publiques (programme d'intervention des agences de financière de bassin...).

Le SAGE sera-t-il au droit de l'eau ce que le S.D.A.U. est à l'urbanisme puisque les décisions relatives aux documents et programmes précités ainsi que les prescriptions édictées au titre de la police de l'eau devront être compatibles? Ainsi, si l'Etat conserve un rôle prépondérant en 
matière de police des eaux, ses pouvoirs en la matière seront exercés dans un cadre déterminé.

1.2. Le principe d'unité de la ressource, s'il n'apporte aucun changement aux statuts des eaux, se manifeste

1.2.1. d'une part, par la substitution à une pluralité de régimes d'une réglementation commune à l'ensemble des eaux indépendamment de leurs caractéristiques physiques et juridiques. Ainsi il y a dépassement des distinctions opérées traditionnellement par le droit : eaux souterraines et eaux superficielles, cours d'eau domaniaux et cours d'eau non domaniaux.

1.2.2. d'autre part, par l'instauration d'une réglementation uniforme pour tous les faits susceptibles d'avoir une incidence sur le milieu aquatique (prélèvements, rejets, ouvrages et travaux...) alors que jusque-là la police des eaux est caractérisée par un enchevêtrement complexe d'autorisations, de déclarations et d'exemptions légales ou réglementaires.

Le système proposé repose sur le principe de l'autorisation - assortie de prescriptions - ou de la déclaration avec des possibilités d'exemption en fonction de l'incidence de l'opération projetée sur le milieu et de la sensibilité de celui-ci.

Le projet réserve toutefois expressément le cas des installations hydroélectriques concédées.

1.2.3. pour finir, en étendant, aux départements d'OutreMer et à Mayotte, tout ou partie des dispositions applicables en métropole.

\section{Une intervention accrue des collectivités territoria- les dans la gestion et la maîtrise des eaux notam- ment fluviales}

2.1. Les communes voient leur obligation s'accroître dans le domaine de l'assainissement, collectif et autonome, pour les eaux usées et les eaux de ruissellement.

2.1.1. Les communes seront tenues d'assurer l'assainissement des eaux usées. Toutefois, cette obligation n'existerait qu'en dehors des zones d'assainissement autonome.

2.1.2. Elles auront aussi l'obligation de délimiter, après enquête publique, les zones d'assainissement autonome qui figureront dans le plan d'occupation des sols. Dans ces zones, les communes seront tenues de mettre en place un service public d'entretien, de contrôle et éventuellement de gestion des dispositifs.

2.1.3. Les communes ou leurs groupements pourront être tenus, dans certains cas, de collecter, stocker voire traiter les eaux pluviales et de ruissellement.
2.2. Les collectivités territoriales et leurs groupements, ainsi que les syndicats mixtes, peuvent être partie prenante en matière de gestion des cours d'eau.

2.2.1. par la maîtrise de travaux, ouvrages ou installations présentant un caractère d'intérêt général ou d'urgence au regard notamment de la sauvegarde des sites, des milieux aquatiques et des zones humides alors même que la collectivité n'a, a priori, aucun droit de propriété ou d'usage.

2.2.2 par la mise en place d'un service public d'entretien régulier de certains cours d'eau non domanial, canaux d'irrigation, de dessèchement ou d'assainissement figurant sur une liste arrêtée par l'Etat.

2.2.3. par le transfert, au profit des collectivités territoriales, qui en font la demande, des compétences pour l'aménagement et l'exploitation des cours d'eau domaniaux rayés de la nomenclature des voies navigables, mais sous réserve de l'existence d'un S.A.G.E. dans le bassin.

2.2.4 par la possibilité d'utiliser la taxe départementale des espaces naturels sensibles pour l'acquisition et la gestion des chemins et servitudes le long des cours d'eau et plans d'eau même non domaniaux.

\section{La nécessité d'assurer un contrôle efficace du respect de la législation par des moyens tels que :}

3.1. l'obligation pour les exploitants, ou à défaut les propriétaires :

3.1.1. de mettre en place des dispositifs de mesures ou d'évaluation pour toute installation de prélèvement et de déversement (sauf exemptions ne pouvant concerner que les prélèvements ou les déversements non soumis à autorisation) ;

3.1.2. d'informer, dans les plus brefs délais, les autorités de police générale (maire ou préfet) en cas d'arrêt d'installations réglementées ou plus largement d'incident ou accident de nature à porter gravement atteinte aux intérêts protégés par la loi. Cette obligation d'information incombe aussi à toute personne à l'origine de l'incident.

Ces différentes personnes doivent en outre prendre toutes les mesures pour mettre fin à la cause de danger ou d'atteinte au milieu aquatique, évaluer les conséquences de l'incident et y remédier.

3.2. la possibilité, pour l'administration, de multiplier les contrôles techniques notamment en donnant un pouvoir de contrôle à un plus grand nombre d'agents et en facilitant leur accès aux installations. 
3.3. le renforcement des sanctions

\subsection{1. pénales}

- par la définition de nouvelles infractions (par exemple : délit de pollution des eaux menaçant la santé publique et d'abandon de déchets, absence d'autorisation ou de déclaration, exploitation d'une installation malgré une mesure de mise hors service ou de suppression),

- par la reconnaissance des pouvoirs importants au profit du juge répressif qui peut ordonner, sous astreinte, des mesures visant à restaurer le milieu aquatique ou à faire cesser l'infraction et suspendre le fonctionnement des installations

- et par la réactualisation du montant des amendes en matière de contravention de grande voirie.

De plus, les associations régulièrement déclarées pourront se constituer partie civile pour les infractions aux dispositions de la loi.

3.3.2. administratives en reprenant les dispositions prévues en matière d'installations classées pour la protection de l'environnement (obligation de pallier les dangers: le préfet peut, après mise en demeure (sauf urgence), prendre ou faire exécuter les mesures nécessaires ; inobservations de la présente loi sanctionnées après mise en demeure par le préfet).

\section{La nécessité de protéger spécifiquement certains usages}

4.1. L'alimentation en eau des populations et plus généralement de la santé et de la salubrité publiques restent prioritaires. Leur protection sera assurée par l'institution, sur demande des collectivités, d'aires de sauvegarde dans laquelle tout prélèvement d'eau sera autorisé à condition de ne pas compromettre les besoins actuels et futurs de l'approvisionnement en eau potable des populations et par l'obligation générale d'instituer des périmètres de protection autour de tous les captages déclarés d'utilité publique - sauf s'ils bénéficient d'une protection naturelle efficace De plus l'avant-projet définit un délit de pollution, analogue à celui protégeant le poisson et son biotope, qui sanctionne toute action ou réaction ayant entraîné, même provisoirement, des effets nuisibles sur la santé, la salubrité publique ou limitant l'usage des zones de baignades.

4.2. La notion de débit affecté subsiste mais a été modifiée. Celui-ci n'est plus attribué à l'Etat mais concédé par l'exploitant d'un ouvrage ayant pour conséquence d'augmenter en période d'étiage ou de régulariser le débit d'un cours d'eau non domanial. L'affectataire du débit verra sa situation protégée puisque l'utilisation du débit affecté par une personne n'y ayant aucun droit sera sanctionnée par une amende et engage sa responsabilité civile vis-à-vis du gestionnaire du débit affecté.

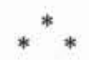

Plus qu'une véritable réforme du droit de l'eau, ces propositions apparaissent plutôt comme une modernisation et une clarification des dispositions existantes afin d'adapter la législation aux nouvelles exigences issues à la fois de la décentralisation et de l'intérêt général qui s'attache à la protection de l'environnement et des ressources naturelles comme l'eau.

On retrouve la logique qui a présidé jusqu'alors à l'élaboration de la réglementation : de nouveaux besoins induisent de nouveaux textes.

De plus, les principes antérieurs n'ont pas été remis en cause : l'administration centrale contrôle toujours, par la délivrance d'autorisations, de récépissé de déclarations et par la définition des exemptions, les différentes actions s'exerçant sur un cours d'eau.

Toutefois, la reconnaissance des écosystèmes aquatiques, la planification des actions dans le cadre du bassin, la généralisation de la police de l'eau et le recours généralisé à la concertation avant décision constituent autant de moyens indispensables pour instituer légalement et pratiquement une véritable gestion des cours d'eau. 


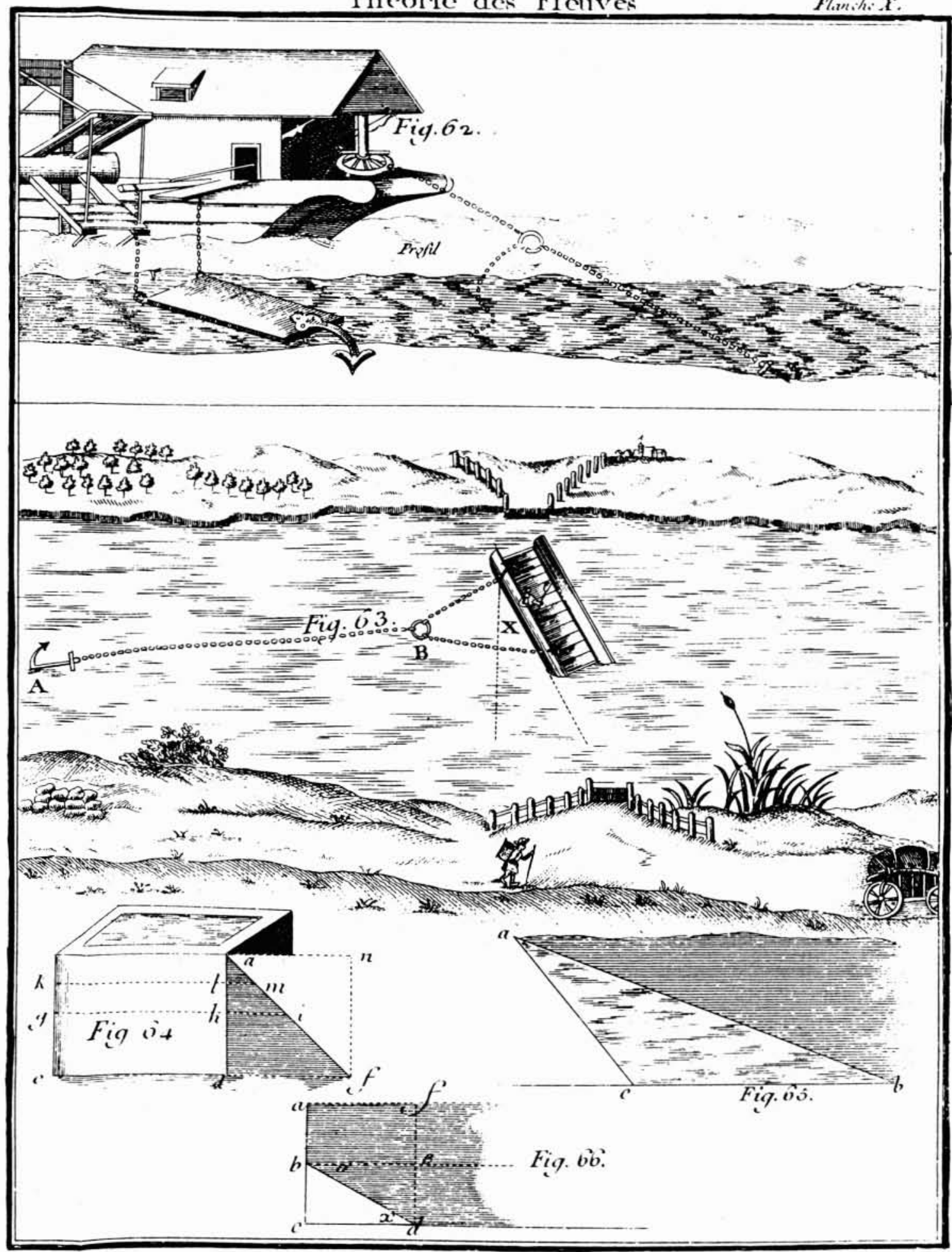

